\title{
Sustainable flexible transport services: a solution against social exclusion
}

\author{
D. Ramazzotti ${ }^{1} \&$ A. Lois ${ }^{2}$ \\ ${ }^{1} S R M$ - Reti e Mobilità, Public Transport Authority of Bologna, Italy \\ ${ }^{2}$ Department of Mechanical and Industrial Engineering, \\ University of Thessaly, Volos, Greece
}

\begin{abstract}
Studies demonstrated that inadequate transport services may create barriers and limit individual and group participation in the normal range of activities. Availability of sustainable transport services can potentially play a very important role in influencing many factors that are enveloped by the concept of social exclusion; in most circumstances inclusion means participation in processes and activities and participation strongly depends on the physical access to facilities.

The right to mobility must be guarantee to mobility-impaired people (children, the elderly and the disabled) even in low demand areas and in the presence of a fragmented public transport service. The Public Transport Authorities (PTAs) can and have to influence and encourage the diffusion of IT based flexible transport systems, being able to link and optimize demand and the offer of transport.
\end{abstract}

Keywords: social and cultural issue, sustainable, flexible transport service.

\section{Introduction}

The right to mobility is a social need and Public Transport Authorities (PTAs) have the mandatory mission to grant it: they are key in the transport chain, because they are able to link demand (citizens and policy-makers) and the offer of transport (operators); furthermore they are skilled in planning, organizing, awarding and controlling services, eventually creating synergies through a webbased platform, hosted and maintained by the PTA itself, where a specific 
working area will be created for each service awarded and it will be possible to book trips, to create vehicle networks optimizing their use (municipal vehicles or school buses out of schedule or taxis), to optimize paths in order to reduce costs and impacts and to monitor services and costs using real-time data.

SRM, in its role of PTA, has set up several contacts with small municipalities in the area of the Province of Bologna. This area has a surface of $3703 \mathrm{~km} 2$, and comprises 59 small cities and a big city (Bologna). The public transport service in the urban area of Bologna is really efficient and punctual (98\%) such as in the main road accessing Bologna. The hill areas south of the city are considered weak demand areas, so public transport there is very poor and infrequent. For this reason the use of private cars is increasing, creating a vicious circle of growing congestion and pollution.

As a public body the PTAs have to answer to these requests. Finding a solution and pilot experiences will be launched in order to help several municipalities (Monte S. Pietro, Borgo Panigale, Grizzana Morandi, etc) in putting their underused transport resources at citizens' disposal, creating synergies between neighbouring areas.

The European Commission also devoted specific attention to less populated areas, as clearly stated in "Keep Europe moving - Sustainable mobility for our continent - Mid-term review of the European Commission's 2001 Transport White Paper": "The outermost regions suffer from a strong accessibility deficit not only in relation to the continental internal market but also in their own hinterland. Transport policy instruments and state aids could be used to reduce the effects of remoteness on their competitive position [...] Action: prepare for the introduction of cooperative systems, to enable co-modal transport solutions and to connect peripheral regions and outermost regions with the mainland; ensure a balanced approach to land use planning."

Participation in the InMosion Project, funded by the EU within the 6th Framework Programme, allowed SRM to conduct analysis and to focus a solution that foresees a new perspective for the PTAs involvement in Italy.

Through a university-based Science Shop (called InMoSion), this project has the overall aim to:

- develop all necessary know-how and expertise to develop an innovative transportation system to meet the mobility needs of elder and mobility challenged Europeans; and

- $\quad$ assist communities with the development and deployment of customized transportation system solutions for their local problems.

To address the specific cost and level of service challenged in moving the elderly population within a society, the proposed system will be a flexible, technologically advanced demand-responsive transport system.

The focus area for a possible pilot experience in Italy was identified in Grizzana Morandi. What has already done in that area will be analyzed later.

The EU project Flipper, funded within the Interreg4C initiative, addresses a key factor of eco-sustainable and competitive development and social cohesion of European areas and Regions, through the investigation, experience exchange, good practices transferring and profitable co-operation on Flexible Transport 
Services (FTS) in relation to mobility in cities, rural areas and small towns. Moreover, Flipper's objective is to achieve capability-building environmental, sustainable and innovative solutions in the Public Transport domain by evaluating viability and real impacts and by gathering the good practices identified at site level.

The challenge of providing a cost effective integrated public transport system is seen as an essential prerequisite to reducing pollution and congestion whilst encouraging sustainable economic growth. The traditional fixed route services is ideal for clustered travel demand areas but the changing of habitation patterns and nature of work activities in today's society have created different mobility needs to which conventional transport has become neither suited nor costeffective.

In the last few years these kinds of services have proved to be an advantageous solution to the provision of public transport services.

In section 2 we describe the basic elements necessary to fully understand the problem. In section 3 a methodology is proposed to solve the problem, starting from the active involvement of PTAs. Section 4 concludes this paper.

\section{Elements definition}

First of all, it is important to quickly define the most important terms of this equation.

\subsection{Social exclusion}

The availability of transport service can potentially play a very important role in influencing many factors that are enveloped by the concept of social exclusion; in most circumstances inclusion means participation in processes and activities and participation strongly depends on physical access to facilities.

Burchardt et al. [1] proposed one of the first quasi-operational definitions: "An individual is socially excluded if (a) he or she is geographically resident in a society and (b) he or she does not participate in the normal activities of citizens in that society" and identify five key dimensions of social exclusion in relation to consumption, savings, production, political and social activities.

It is known that the elderly and disabled comprise a vital part of our society and have a lot to contribute, if they have the ability to travel easily and to access major attractions and activity locations in a neighbourhood.

Talking about children we have to consider their impossibility to move alone using private means. The access to education, health care and leisure activities is important for them as well in order to ensure their involvement and their development.

The availability of public transport services, in particular in deprived or low density peripheral areas, is a vital link to access to urban facilities and services. The provision of tailored travel solutions adapted to the needs of specific groups is a useful complement to conventional public transport services [2]. 


\subsection{Sustainable transport}

A first definition came from a publication of the United Nations "Our Common Future" [3] where took its origin the concept of sustainable development. Descending from that, "Sustainable transportation is about meeting or helping meet the mobility needs of the present without compromising the ability of future generations to meet their needs". The main aim is reducing fuel consumption and polluting emissions.

A further definition by the World Business Council for Sustainable Development starts referring to "sustainable mobility" defining it as the "ability to meet the needs of society to move freely, gain access, communicate, trade and establish relationships without sacrificing other essential human or ecological values today or in the future" [4].

A necessary and complementary action to allow sustainability refers to the effort to reduce the impact of transportation in terms of environmental pollution and energy consumption increasing efficiency and efficacy through the transport demand management.

\subsection{Flexible Transport Services (FTSs)}

FTS are complementary to the conventional passenger transport and usually serve no scheduled mobility needs, either in low demand time periods and in weak demand areas. Given this flexibility, FTS are suitable to serve niche market customers and different citizens' groups (e.g. disabled and elderly, students, tourists, etc.). FTS usually operate with small size buses on flexible routes and scheduling, based on the citizens needs and requests (off-peak hours, low demand zones, disadvantaged citizens like elderly and disabled, etc.).

Since these kinds of systems are mainly needed in areas with low infrastructures and low investment capacity, these kinds of transport services may be configured as:

- alternative to regular public transport (traditional) in order to rationalise the use of the resources and consequently reduce pollution and the costs by adapting the service to the real needs of the users;

- integrations to the regular service, fitting in a presumably higher quality range and guaranteeing furthermore an extra/distribution function to backup the leading service;

Flexibility in transport services can be referred to:

- route/path;

- schedule;

- type of vehicle;

- combination of the above elements.

\section{Proposed methodology to solve the problem}

The problem is to provide a "non traditional" transportation service to these isolated areas. Because of the nature of that transportation service a specific 
methodology is needed; it is necessary to define setup procedures, to make IT platforms available, to agree measurement and evaluating procedures in order to clarify if the proposed system is worth or not. The PTAs can play a very interesting role as a key chain ring, linking the citizens needs, represented by the relative municipalities, and the transport offer, that needs to be harmonised and optimized to obtain synergic effects and resources optimization. They can also support decision makers with specific data and surveys, prepare service tendering procedures, offer easy access to the system, support and control the service providing, monitor and evaluate the pilot results at a service level and at a customer satisfaction level in order to decide if the service is sustainable from the different points of view.

\subsection{Methodology basic steps}

The main phases to create a new transport service, flexible and innovative, are:

Step 1 : service identification;

Step 2 : service setting up defining network, fares, vehicles, timetables, quality standards, IT application;

Step 3 : service awarding, if needed, and contractual matter including: regulations, accessibility, vehicles, fares, penalty (quality);

Step 4 : IT platform availability;

Step 5: evaluation of the service with qualitative and quantitative measurement, including controls on operators activities;

Step 6: PTA contribution to the service for a first year of exploitation with own funds.

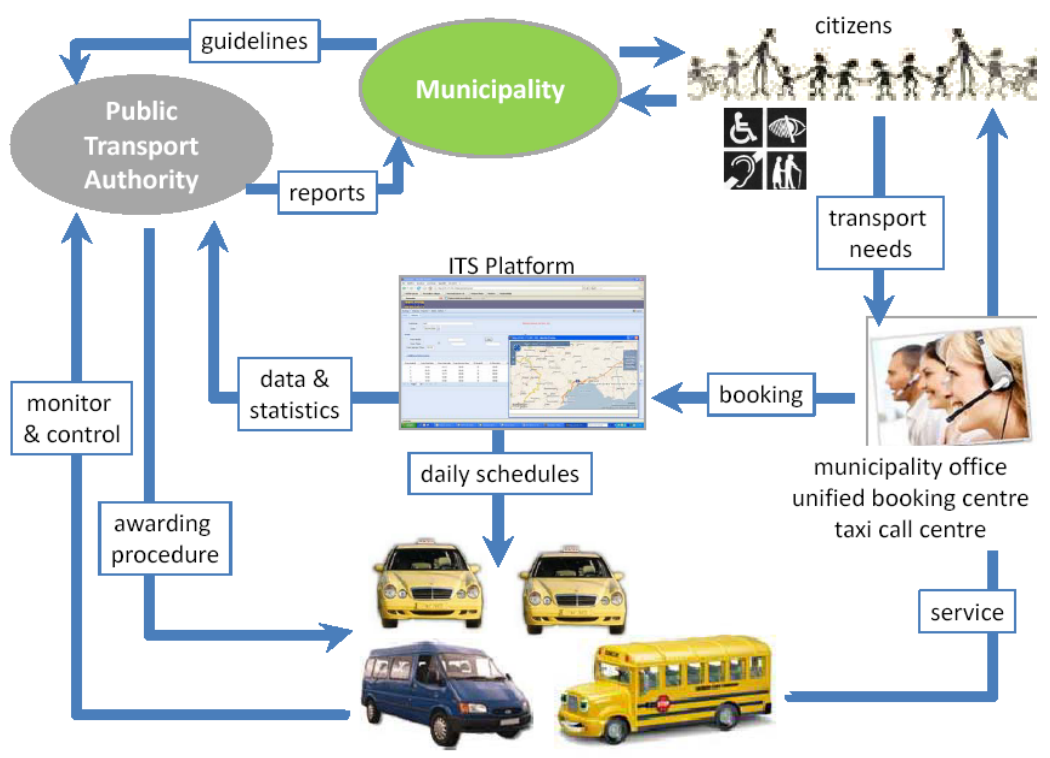

Figure 1: Organizational scheme for FTS supervised by PTA. 


\subsection{Service identification}

A deep analysis should be conducted in order to have a first evaluation of:

- Area and population profile: clearly identify the area to be covered and study the demographic and socio-economic profile (i.e. age, structure, unemployment, car ownership, transport habits).

- Attraction points: basic essential services in the area have to be tracked in order to identify the most suitable origin and destination points: schools, health care structures, access to railway or high frequency public transport, commercial, etc.

- Population needs: identify transport needs for different groups like commuters, children, youth, elderly and people with disabilities).

- Legal framework: it is necessary to conduct a deep analysis of the existing laws and rules.

- Transport services existing in the area: if we talk about rural or mountain areas, existing transport services are usually poor in terms of lines and frequency; if we talk about elderly or people with disabilities we can focus also on urban areas: the regular existing transport vehicles are not suitable for this group of users.

\subsection{Test case}

The first three points can be investigated through a survey. In Grizzana Morandi (Italy) example the survey was distributed by Mayor offices to all pupils and students.
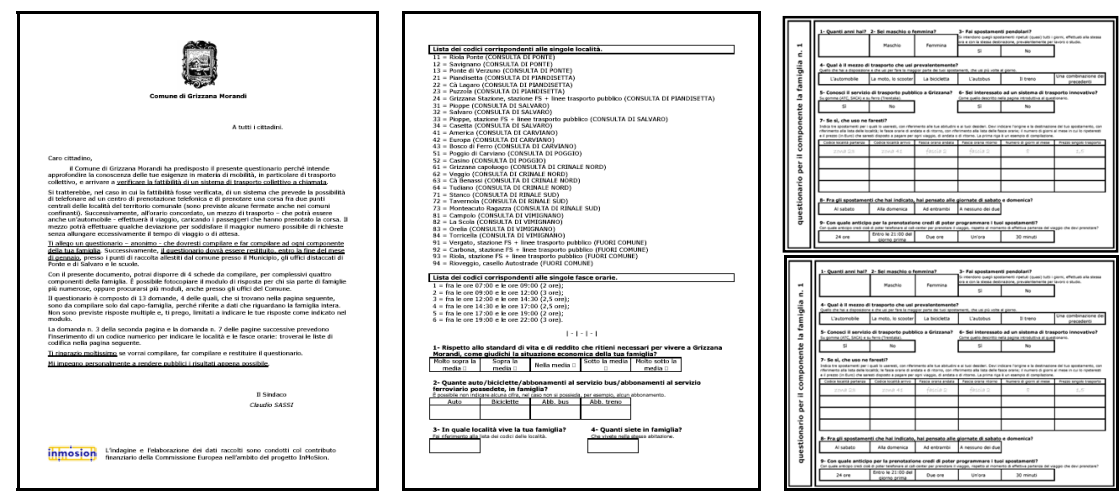

Figure 2: $\quad$ Survey distributed in Grizzana Morandi. A letter from the mayor was in the first page, in the second page a little data about family habits and conditions were requested and there are four parts for individual answers that need indication.

From the survey we could extract the elements needed to prepare a feasibility study and to plan the needed service: 
- 58\% of interviewed citizens expressed their interest for a demandresponsive transport service, whose main features were presented by the mayor in the first page of the questionnaire: one citizen in two expressed interest in such kind of service;

- this interest is strongest amongst citizens that use more than a mean $(80 \%)$, county town residents $(70 \%)$ and regular commuters $(65 \%)$;

- the use of the service results interesting for an average number of 15 days a month. In $75 \%$ of cases the citizens expressed interest for the service if extended to Saturday and Sunday;

- the matrix of the time slots underlines a widespread use, not concentrated in peak or weak hours. In particular the one-way trip are concentrated $60 \%$ in time slot 7:00-9:00 and the back trips are divided, with different percentage, in the afternoon slots 12:30-14:30, 14:30-17:00, 17:00-19:00 and 19:00-22:00;

- the minimum advance time to book the service was quantified: only $20 \%$ of population sets 30 minutes before; accepting booking within 21:00 of the evening before three customers each five will be satisfied;

- the average price the interviewed said are ready to pay for the service is $1,25 € /$ trip. Setting up the fare at $1,00 € /$ trip without taking in account the distance, the $90 \%$ of the potential customers will be satisfied.

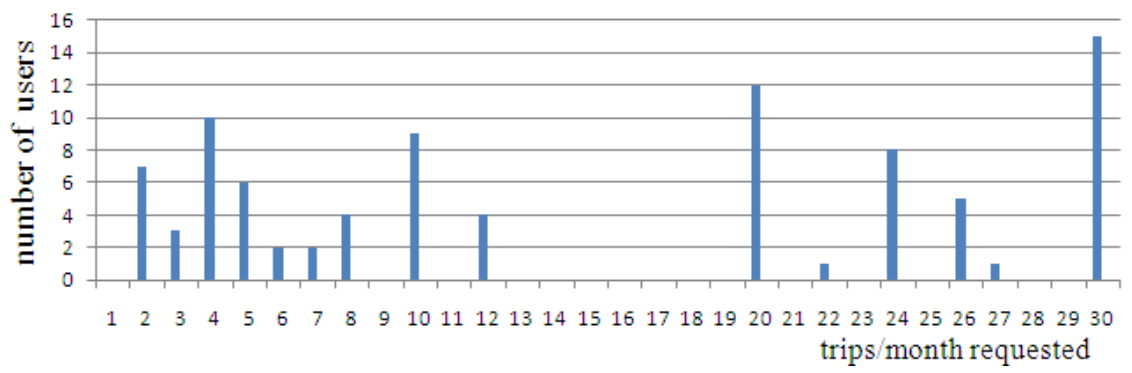

Figure 3: Number of days a month requested for the service.

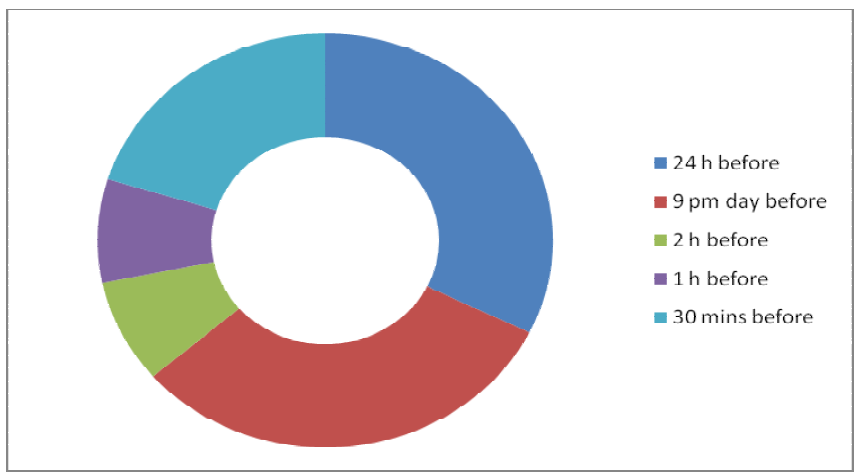

Figure 4: Advanced time booking availability. 


\subsection{Service setting up (by competent public bodies)}

After conducting the survey and the results analysis, the authority can act as a consultant for the local public body (municipality, county, etc) that has to authorize the new service.

SRM also conducted a deep analysis of the Italian legal framework for highly innovative and flexible local public transport services.

The Italian framework is very complex but we highlighted that to identify the service as "public" we need:

- access also without booking;

- to be functional and integrated with leading service;

- fare integration.

Analyzing the existing Italian legal and administrative framework is evident that laws and rules in the transport world are often obsolete and do not lend themselves to the introduction of new ideas, strategies, and practices. Only small windows of opportunity are available for other bodies or local administrations to operate flexible transportation systems. The PT Authority has the competence and the skills to set up a service fully compliant to rules and answering the users' needs.

\subsection{Service awarding, if needed, and contractual matters including: regulations, accessibility, vehicles, fares, penalty (quality)}

The PTA, on behalf of the competent local body, will award the service or tendering it, depending on the local body guidelines and on the law.

In general, FTS are services with small quantity of kilometres and they can often be considered as an integration of existing service contracts, included in the so called "jus variandi", as to say in the flexibility foreseen by the contract.

PTA will also include all contractual provisions that the operator should respect in running the service.

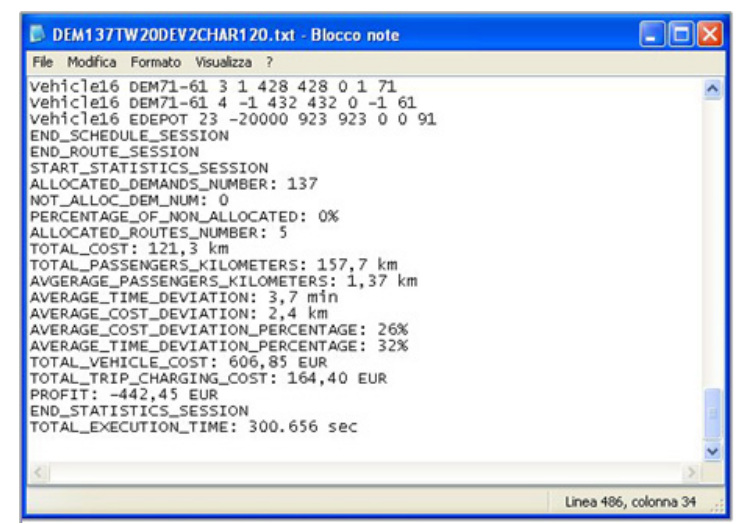

Figure 5: Simulation of a service in order to evaluate its sustainability. 


\subsection{IT platform availability}

IT based procedure will also allow to the PTA to have direct access to data about the service, useful to evaluate operator performances, to control them and to intervene with "real-time" decisions. Furthermore special features are available to perform simulation under different scenarios, with defined and given input, to help evaluation about fares and vehicle capacity, including economic point of view.

In the case of SRM, the PTA of Bologna, Italy, the platform has been developed by the University of Thessaly within the framework of the EU project InMoSion (Science Shop for Innovative Mobility Solutions for Mobility Challenged Europeans).

The InMoSion software is already available, it has been tested in a 45-day pilot service in Greece and it is under test phase for an Italian pilot in BolognaBorgo Panigale, which will start in March 2009.

SRM, as PTA decide to adopt this approach and became a local platform owner. SRM will allow the access to the software to the operator of flexible transport services in its area, creating different separated networks (with given stops and rules descending by the contract) and a system of protected access with username and passwords.

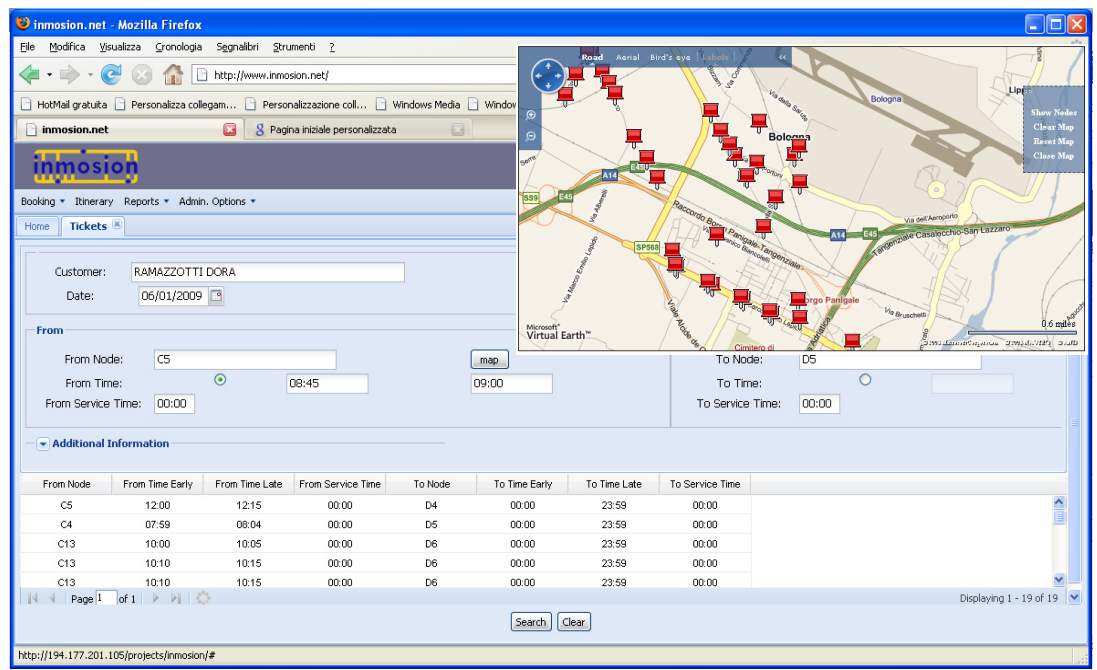

Figure 6: Booking interface of the InMoSion system [5].

Flexible services are often run by small operators, who cannot afford to buy and maintain expensive optimization software.

A dedicated software has been developed to book trips but the most important tool is the algorithm that elaborate and optimize the passengers' requests in order to reduce kilometres, emissions and therefore costs.

The algorithms were used in an innovative combination: the initialization step of the algorithm initializes the system by sorting the trip demands and the set of 
vehicles and does the appropriate trip demand allocation to the route, ensuring that the additional cost of the insertion is the smallest; the second algorithm is an Inter-route algorithm that runs continuously and optimizes every route by first removing the allocated trip demand from the route it belongs and then reinserting it into a better position in another route in terms of decreasing Total Route Cost.

It was demonstrated that the approach produces results very close to the optimal solution. The algorithm can be used as the core engine in a flexible transport scheduling system, where is critical to provide good routes in order to provide better customer service and kilometre reducing.

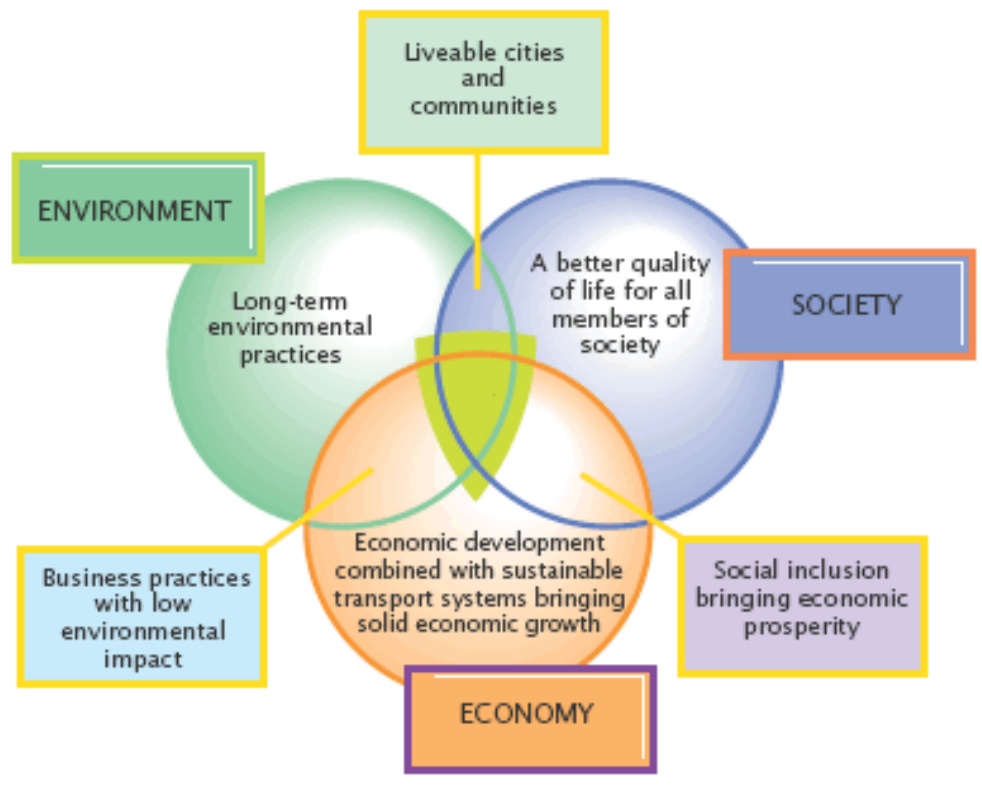

Figure 7: Economic, social and environmental objectives are considered together.

\subsection{Evaluation of the service with qualitative and quantitative measurement, including controls on operators activities}

In order to evaluate the sustainability of a transport system, different sets of indicators can be used. It is important to keep in mind that the introduction of such systems influences the social, economic and environmental aspects of a community.

In particular a comprehensive approach can be very useful to identify strategies for integrated planning to achieve win-win solutions. [6]:

After the pilot exploitation, for an IT based FTS the performance measures can be done referring directly to data, with no need of further data collection or operator forecast, looking for efficiency, effectiveness and quality [7]. The most used indicators are related to the following. 


\subsubsection{Efficiency}

Average number of passengers per vehicle

Average number of passengers-km per vehicle

Average operating speed (km/hour)

Average percentage waiting time

Average percentage unloaded $\mathrm{km}$

Costs of intake, planning and dispatch per trip

Costs of intake, planning and dispatch per trip related to operating costs

Percentage overhead costs related to total costs

\subsubsection{Effectiveness}

Number of passenger trip per year

Number of passenger-kilometres per year

Number of passenger trips per inhabitant

Number of passenger trips per person in a target group

\subsubsection{Quality}

Image Customer satisfaction (can be measured upon passengers feedback) Percentage trips in time/within quality parameters (measures with IT tools)

The value of this parameters can be prefixed by the local public body in agreement with the authority to define the satisfactory exploitation; the indicators and the value can be widely different depending on the policy-maker strategies and the objective features of the specific system (inhabitants, type of vehicle, road network, target groups, etc).

\subsubsection{PTA contribution to the service for a first year of exploitation with own funds}

The Board of Administration of SRM, supported by the ownership (Municipality and Province of Bologna) took the decision to assign specific contributions to pilot experiences of FTS. A fund of 125.000 euro has been foreseen in 2007 and will be doubled in 2008 for this purpose.

The interested municipalities can ask for Authority's support in all the preliminary phases and also for the first year of experimental exploitation taking the obligation to continue the service if the evaluation after a year will be positive.

Several examples and data of positive experiences of FTSs were analyzed and collected by the Mascara EU project [8].

In the conclusive analysis it is highlighted that the global value presented should be viewed with caution, as it is a sum of values estimated taking into account the local, regional points of view. Nevertheless, it is clear that, when taking into account indirect non-monetary benefits, FTSs are capable of demonstrating a positive net return.

\section{Conclusions}

In the paper we identified that the problem of social exclusion is often due to a physical impossibility to take part in social life. Citizens that live in weak 
demand areas and disabled people have mobility needs that remain often unsatisfied by existing transport because this kind of service it is not sustainable from economical and environmental point of view. FTS, on the opposite, is able to answer real needs increasing therefore efficiency, efficacy and customer satisfaction.

The barriers that a small municipality can face in establishing such services can be crossed when a PTA puts at disposal its skills and its know-how. The use of available IT tools has been extensively tested and allows the management of a service granting optimization at all levels, including use of resources and costs. This innovative way to proceed allow to make very clear to the population that the specific line is in a test phase and that after one year of exploitation the results will be evaluated: this will be a guarantee that if a system is not sustainable, the municipality will not waste further money; if a system satisfy passengers needs and number of trips grows consistently the FTS line can be "upgraded" to a regular transport line, knowing exactly passengers needs thanks to reservation trend and adapting therefore the bus schedule.

The support, also economical, of PTAs can strongly encourage the diffusion of flexible transport systems that are a real step ahead in the fight against social exclusion.

\section{References}

[1] Burchard, T., et al. Degrees of Exclusion in John Hills et al. Understanding Social Exclusion, Oxford University Press, 2002.

[2] Magarth, T., Where there is mobility there is social inclusion, UITP Public Transport International, 2008.

[3] United Nations, World Commission on Environment and Development, Our Common Future A/42/427, 1987.

[4] WBCSD - World Business Council for Sustainable Development, Mobility 2001 - World mobility at the end of the twentieth century and its sustainability Report, 2001.

[5] InMoSion website, www.inmosion.net

[6] UITP Public Transport International, Ticket for tomorrow (source Ralph Hall, Introducing the Concept of Sustainable Transport), 2008.

[7] Project CONNECT, Position paper on Flexible Transport Systems Business Model, 2005.

[8] Project MASCARA, Final Evaluation Report-Volume 4-Evaluation tool Results, Cost-Benefit analysis, 2008. 